\section{How Much Force Is Needed To Stretch a Coiled Chain in Solution?}

\author{
Hui Ge, ${ }^{\dagger}$ Fan Jin, ${ }^{\dagger}$ Junfang $\mathrm{Li}^{*}{ }^{*}$ and $\mathrm{Chi} \mathrm{Wu}^{*}{ }^{, \dagger}, *$ \\ ${ }^{\dagger}$ Department of Chemistry, The Chinese University of Hong \\ Kong, Shatin, N.T., Hong Kong, and The Hefei National \\ Laboratory of Physical Science at Microscale, Department of \\ Chemical Physics, The University of Science and Technology of \\ China, Hefei, Anhui 230026, China
}

Received March 14, 2009

Revised Manuscript Received May 27, 2009

In the 1970s, de Gennes and Pincus ${ }^{1,2}$ used the Rouse model ${ }^{3}$ to show that linear polymer chains in a good solvent could undergo a first-order coil-to-stretch transition to pass through a pore much smaller than its coiled size under an elongation flow field with a sufficient hydrodynamic force, independent of both the chain length and the pore size. Namely, a coiled polymer chain can pass through a small pore as long as the flow rate $(q)$ is higher than a threshold $\left[q_{\mathrm{c}} \simeq k_{\mathrm{B}} T /(3 \pi \eta)\right]$, where $k_{\mathrm{B}}, T$, and $\eta$ is the Boltzmann constant, the absolutely temperature, and the solvent viscosity, respectively.

On the other hand, it has been suggested that the electrostatic blob model for a polyelectrolytes chain would also be applicable for the coil-to-stretch transition of a neutral chain in an ultrafiltration experiment. ${ }^{4,5}$ In principle, the stretching of a neutral chain under an elongational flow and the extension of a polyelectrolyte chain under the electrostatic repulsion have a similar physical nature, but not identical. Namely, a neutral chain can be stretched as a string of blobs under an elongational flow. The blob size decreases as the shear rate increases. Finally, each blob can be fully stretched with a sufficiently strong shear force.

It is not difficult to realize that the passing through a small pore occurs only when the blob size $\left(\xi_{\mathrm{b}}\right)$ of a stretched chain in a flow filed is smaller than the pore size $(D)$. Therefore, the critical flow rate is associated with the relative size of the blob to the pore. Whether a coiled chain can pass through a small pore is related to its local deformability, i.e., the entering of the first blob into the pore, at which the entropic confinement force $\left(k_{\mathrm{B}} T / D\right)$ is overcame by the hydrodynamic force $\left(3 \pi \eta q_{\mathrm{c}} / D\right)$, where it has assumed that each blob is a nondraining ball with a diameter identical to the pore size $(D) .{ }^{1,2}$ Note that here only the short-range interaction is relevant. In principle, one can reversibly use $q_{\mathrm{c}}$ to characterize the local deformability. To our knowledge, the predicted chain-length and pore-size independence of $q_{\mathrm{c}}$ has not been confirmed in experiments because such a first-order coil-tostretch transition has not been observed for a long time. Recently, we have observed such a discontinuous first-order transition by ultrafiltrating linear polystyrene chains through specially constructed small pores $(20 \mathrm{~nm}){ }^{6}$

Note that the rigidity of polymer chain has been traditionally characterized in terms of the persistence length $\left(l_{\mathrm{p}}\right)$, an important parameter not only in polymer research but also in the study of protein unfolding under an exerted force..$^{7-9}$ Normally, $l_{\mathrm{p}}$ is deduced from the measured average radius of gyration $\left(\left\langle R_{\mathrm{g}}{ }^{2}\right\rangle^{1 / 2}\right) .{ }^{10}$ For a more rigid chain, ${ }^{11} l_{\mathrm{p}}$ can be indirectly estimated

\footnotetext{
*Corresponding author. The Hong Kong address should be used for all correspondence.
}

from the intrinsic viscosity $\left([\eta]_{\theta}\right) .{ }^{12}$ Also note that the developments of electron microscopy ${ }^{13,14}$ and $\mathrm{AFM}^{15-17}$ lead to some direct measurements of $l_{\mathrm{p}}$ in terms of the conformation change and the force required to extend a polymer chain. It should be emphasized that a combination of the magnetic tweezer and hydrodynamic force can also be used to measure the weaker entropy elasticity of a long and more rigid DNA chain. ${ }^{18}$

In the current ultrafiltration experiments, we find $q_{\mathrm{c}}$ from the abrupt change of the relative retention $\left[\left(C_{0}-C\right) / C_{0}\right]$ of linear chains, where $C_{0}$ and $C$ are polymer concentrations respectively before and after the solution passes through smaller pores. At this critical flow rate, the hydrodynamic force starts to overcome the entropic confinement force. Therefore, we can estimate how much force is needed to stretch a coiled chain into a string of blobs with a size of $D$. In this study, the solution temperaturedependent ultrafiltration of linear polystyrene (PS) chains in cyclohexane was measured by using the Whatman membranes with two different pore sizes $(20$ and $100 \mathrm{~nm})$. The average pore density is $5 \times 10^{8}$ per membrane, and the length of each pore is $1 \mu \mathrm{m}$. The membranes have a double-layer structure that prevents some possible interference among flow fields generated by different pores, leading to an elongation one as required by the theory.

In order to increase the accuracy, we used a solution mixture of two PS samples with different chains lengths; namely, long chains (PS-L, $M_{\mathrm{w}}>2.2 \times 10^{6} \mathrm{~g} / \mathrm{mol}, M_{\mathrm{w}} / M_{\mathrm{n}}<1.10$, and $\left\langle R_{\mathrm{h}}\right\rangle>25 \mathrm{~nm}$ ) and short chains $\left(\mathrm{PS}-\mathrm{S}, M_{\mathrm{w}}=4.4 \times 10^{4} \mathrm{~g} / \mathrm{mol}, M_{\mathrm{w}} / M_{\mathrm{n}}=1.13\right.$, and $\left.\left\langle R_{\mathrm{h}}\right\rangle=5 \mathrm{~nm}\right)$. Note that there is no retention for short PS chains at any flow rate. The solution mixture of PS-S $\left(C_{\text {PS-S }}=1.00 \times 10^{-2}\right.$ $\mathrm{g} / \mathrm{mL})$ and PS-L $\left(C_{\text {PS-L }}=(2.4-20.0) \times 10^{-5} \mathrm{~g} / \mathrm{mL}\right)$ with a volume ratio of $\sim 1: 1$ was used as the stock solution. In each ultrafiltration experiment, the macroscopic flow rate $(Q)$ and temperature were controlled by a syringe pump (Harvard Apparatus, PHD 2000) and an incubator (Stuart Scientific, S160D) $\left( \pm 0.1^{\circ} \mathrm{C}\right)$, respectively. In our setup, at the lowest used flow rate of $0.005 \mathrm{~mL} / \mathrm{h}$, the linear moving rate is $3.56 \times 10^{-6} \mathrm{~cm} / \mathrm{s}$. The minimum linear moving rate of the pump (motor) is $3 \times 10^{-7} \mathrm{~cm} / \mathrm{s}, 10$ times smaller than the rate used. Therefore, the flow used can be treated as a continuous one. A combination of static and dynamic laser light scattering (LLS) measurements enables us to accurately determine the relative retention concentration of longer chains under different macroscopic flow rates by using short chains as an internal reference. ${ }^{6}$

Figure 1 shows how the measured $\left(C_{0}-C\right) / C_{0}$ depends on the flow rate $(Q)$ for polystyrene chains with different lengths in cyclohexane, where the pore diameter $(D)$ is $20 \mathrm{~nm}$. It clearly shows that there is no retention for the shortest chain because its size is smaller than the pore size, while for the longer chains, the retention nearly starts at an identical flow rate $\left(\sim 2 \times 10^{-2} \mathrm{~mL} / \mathrm{h}\right)$ in spite of their different lengths. Such a critical flow rate or threshold for long PS chains to pass through smaller pores is welldefined because the coil-to-stretching is a first-order transition. ${ }^{2}$ In this way, we were able to accurately measure the critical flow rate $\left(Q_{\mathrm{c}}\right)$ of longer PS chains through small pores.

On the other hand, it has been known that for long PS chains in cyclohexane the theta temperature $(\Theta)$ is $\sim 34.5^{\circ} \mathrm{C}$, ${ }^{19}$ at which the chains behave like a Gaussian coil. ${ }^{20}$ Figure 2 shows that the light scattering measured average radius of gyration $\left(\left\langle R_{\mathrm{g}}\right\rangle\right)$ of long PS chains $\left(M_{\mathrm{w}}=1.8 \times 10^{7} \mathrm{~g} / \mathrm{mol}, M_{\mathrm{w}} / M_{\mathrm{n}}=1.06\right.$, and $\left.\left\langle R_{\mathrm{h}}\right\rangle=100 \mathrm{~nm}\right)$, specially synthesized by high-vacuum anionic polymerization, monotonely decreases with the solution temperature, 


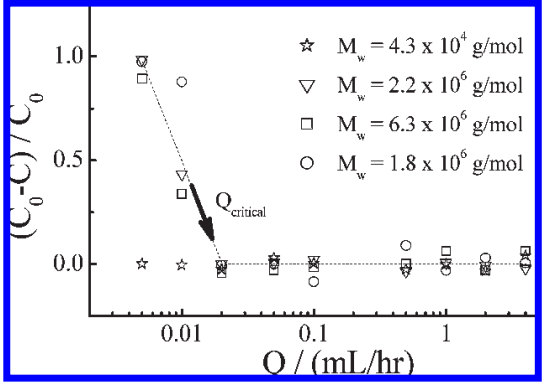

Figure 1. Macroscopic flow rate $(Q)$ dependence of retention concentration of different molecular weights PS linear chains in cyclohexane at $T=34.5^{\circ} \mathrm{C}$

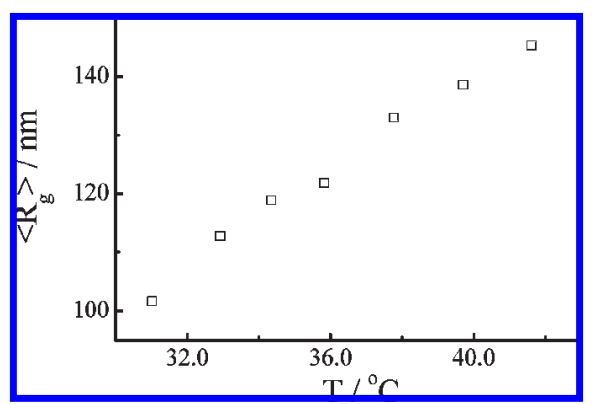

Figure 2. Solution temperature dependence of average radius of gyration $\left\langle R_{\mathrm{g}}\right\rangle$ of long polystyrene chains (PS-L) in cyclohexane.

corresponding to a volume decrease of three times. As expected, the chain deformation at different temperatures should be different because of their different swelling degrees. At higher temperatures, it would require a stronger force to overcome the entropic force to deform a given chain. On the other hand, when the chain is collapsed in a poor solvent with stronger segmentsegment interaction, the force required to deform it would also be higher.

Figure 3 shows passing or nonpassing of long PS chains through small pores $(20 \mathrm{~nm})$ at different temperatures. For a given number of small pores on each membrane $(N)$, we can convert the macroscopic flow rate $(Q)$ into the average microscopic flow rate $(q=Q / N)$ and the average flow velocity $(v=$ $\left.q / D^{2}\right)$ inside each pore. As discussed before, $q_{\mathrm{c}}$ is directly related to the hydrodynamic force $\left(f_{\mathrm{h}}\right)$ required to stretch a coiled polymer chain in solution by $f_{\mathrm{h}}=3 \pi \eta\left(q / D^{2}\right) L_{\mathrm{e}}$, where $L_{\mathrm{e}}$ is the effective length along the flow direction. For a nondraining hard ball with a diameter of $D, L_{\mathrm{e}}=D$. For a given pore size, our experimental results show that $q_{\mathrm{c}}$ remains a constant, $5.56 \times$ $10^{-15} \mathrm{~mL} / \mathrm{s}$ at $T=\Theta$, for the three PS samples measured, independent of the chain length as predicted by de Gennes. ${ }^{21}$

However, our measured $q_{\mathrm{c}}$ values are $\sim 10-200$ times smaller than $k_{\mathrm{B}} T /(3 \pi \eta)$ predicted. ${ }^{1,2}$ Note that in the prediction the confinement force $\left(f_{\mathrm{c}}\right)$ equals $\left(k_{\mathrm{B}} T / D^{3}\right) D^{2}$, i.e., $f_{\mathrm{c}}=k_{\mathrm{B}} T / D$, where $k_{\mathrm{B}} T / D^{3}$ is the osmotic pressure generated by the random Brownian motion of the gravity center of the chain segments inside each blob. ${ }^{21,22}$ In reality, each blob contains a swollen chain segment and is draining; namely, the hydrodynamic force $\left[f_{\mathrm{h}}=3 \pi \eta\left(q / D^{2}\right) L_{\mathrm{e}}\right]$ on each blob should be much stronger than $3 \pi \eta q / D$ because $L_{\mathrm{e}} \gg D$. For a fully draining blob made of $n$ segments and each with an average length of $l, L_{\mathrm{e}}=\ln$ and $n=M_{\mathrm{b}} / M_{0}$, where $M_{\mathrm{b}}$ and $M_{0}$ are the molar masses of the blob and the segment, respectively. When $f_{\mathrm{h}}=f_{\mathrm{c}}, q$ reaches its critical value, i.e.

$$
q_{\mathrm{c}}=\frac{k_{\mathrm{B}} T}{3 \pi \eta}\left(\frac{D}{L_{\mathrm{e}}}\right)
$$

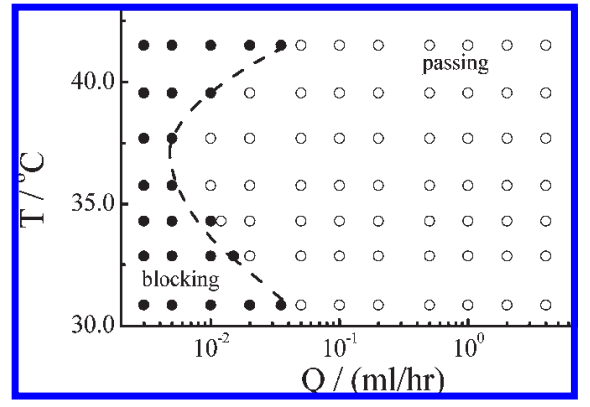

Figure 3. Phase diagram of passing or blocking of long PS-L chains in cyclohexane through small pores $(20 \mathrm{~nm})$ at different solution temperatures, where hollow and filled symbols respectively represent passing and blocking.

Table 1. Solution-Temperature Dependence of Critical Macroscopic Flow Rate $\left(Q_{\mathrm{c}}\right)$, Reduced Critical Microscopic Flow Rate $\left(q_{\mathrm{c}} /\left(k_{\mathrm{B}} T\right)\right.$ $\eta)$ ), and Estimated Hydrodynamic Force $\left(f_{\mathrm{h}}\right)$ Required To Deform, Stretch, and Pull Long Linear Polystyrene Chains in Cyclohexane To Pass through Small Pores (20 nm $)^{a}$

\begin{tabular}{llllllll}
\hline$T /{ }^{\circ} \mathrm{C}$ & 30.85 & 32.85 & 34.30 & 35.80 & 37.70 & 39.55 & 41.55 \\
$Q_{\mathrm{c}} / 10^{-2} \mathrm{~mL} \cdot \mathrm{h}^{-1}$ & 4.25 & 1.50 & 0.75 & 0.60 & 1.10 & 1.75 & 4.25 \\
$q_{\mathrm{c}} /\left[k_{\mathrm{B}} T /(3 \pi \eta)\right] / 10^{-2}$ & 4.32 & 1.47 & 0.72 & 0.56 & 0.99 & 1.52 & 3.56 \\
$f_{\mathrm{h}} / \mathrm{fN}$ & 144 & 58 & 35 & 19 & 20 & 38 & 101
\end{tabular}

${ }^{a} f_{\mathrm{h}}$ is calculated using eq 3 (please refer the text for details), and the uncertainties of $Q_{\mathrm{c}}$ (or $q_{\mathrm{c}}$ ) and $f_{\mathrm{h}}$ are $\pm 5 \%$ and $\pm 10 \%$, respectively.

Qualitatively, it explains why our measured $q_{\mathrm{c}}$ values are much smaller than $k_{\mathrm{B}} T /(3 \pi \eta)$ because $L_{\mathrm{e}} \gg D$. Quantitatively, we can estimate $L_{\mathrm{e}}$ assuming that each blob has a size of $D$. Using the scaling $D=k M_{\mathrm{b}}{ }^{\alpha}$, we have $L_{\mathrm{e}}=l M_{\mathrm{b}} / M_{0}=\left(l / M_{0}\right) k^{-1 / \alpha} D^{1 / \alpha}$, where $\alpha$ is the Flory exponent and $k$ is a constant for a given polymer solution. Equation 1 can be rewritten as

$$
q_{\mathrm{c}}=\frac{k_{\mathrm{B}} T}{3 \pi \eta}\left(\frac{M_{0}}{l}\right) \frac{D}{M_{\mathrm{b}}}=\frac{k_{\mathrm{B}} T}{3 \pi \eta} \frac{M_{0}}{l} k^{1 / \alpha} D^{1-(1 / \alpha)}
$$

It explains why $q_{\mathrm{c}} /\left[k_{\mathrm{B}} T /(3 \pi \eta)\right]$ decreases with the temperature in the range $T \geq \Theta$ because $M_{\mathrm{b}}$ increases as the chain shrinks for a given pore size $(D)$. Quantitatively, taking the theta state as a reference, ${ }^{23}$ we have $k=2.82 \times 10^{-9} \mathrm{~cm}$ and $\alpha=0.5$. Replacing $M_{0}=104 \mathrm{~g} / \mathrm{mol}, D=2 \times 10^{-6} \mathrm{~cm}$, and $l \approx 0.26 \mathrm{~nm}=2.6 \times 10^{-8} \mathrm{~cm}$, we have $q_{\mathrm{c}}\left[\left[k_{\mathrm{B}} T /(3 \pi \eta)\right] \approx 1.6 \times 10^{-2}\right.$, not too far away from those listed in Table 1. Following the same argument, we can estimate the hydrodynamic force on one blob by using

$$
f_{\mathrm{h}}=3 \pi \eta \frac{q_{\mathrm{c}}}{D^{2}} L_{\mathrm{e}}=\frac{3 \pi \eta l}{M_{0}} q_{\mathrm{c}} k^{-1 / \alpha} D^{-2+(1 / \alpha)}
$$

Recently, Melchionna et al. ${ }^{24}$ showed that under a strong squeeze force the chain could self-order into a multifolded state inside a small pore. Here the hydrodynamic force used is very weak and only overcomes the entropic elasticity. Therefore, it is reasonable to assume that the chain inside the pore is still made of a string of blobs and the subchain inside each blob follows Gaussian statistics. On the other hand, $\alpha$ is weakly dependent on the temperature in the range studied anyway. Using $T=\Theta\left(34.5^{\circ} \mathrm{C}\right)$ as a reference point, we can estimate $k$ and $\alpha$ in the range $T \geq \Theta$ from Figure 2, wherein $M$ is a constant, and then calculate the temperature dependence of $f_{\mathrm{h}}$, as shown in Figure 4 and also listed in Table 1, where $\tau=(T-\Theta) / \Theta$.

Figure 4 shows that $f_{\mathrm{h}}=1.0 \times 10^{-13} \mathrm{~N}$ at $\sim 42{ }^{\circ} \mathrm{C}$, not too far away from the calculated confinement force $\left(k_{\mathrm{B}} T / D \sim 2.17 \times\right.$ $\left.10^{-13} \mathrm{~N}\right)$. Note that $f_{\mathrm{h}}$ reaches a minimum at $\sim 36.5^{\circ} \mathrm{C}$, slightly away from the theta point $(\tau=0)$. This is not a surprise because $\Theta$ 


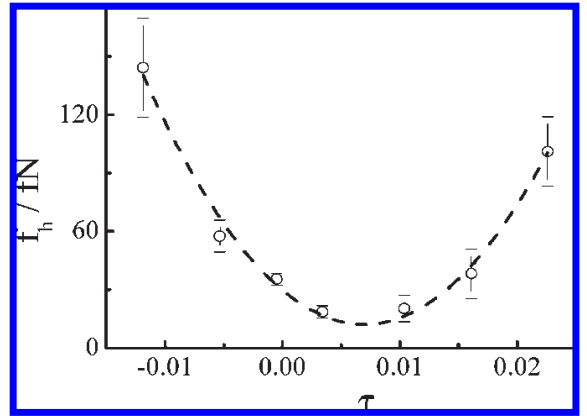

Figure 4. Solution temperature dependence of hydrodynamic force $\left(f_{\mathrm{h}}\right)$ needed to pull coiled linear polystyrene chains in cyclohexane through small pore $(20 \mathrm{~nm})$, where $\tau=(T-\Theta) / \Theta$, a reduced solution temperature, and $\Theta$ is a solution temperature at which the second virial coefficient vanishes.

Table 2. Pore Size Dependence of Critical Macroscopic Flow Rate $\left(q_{\mathrm{c}}\right)$ and Estimated Hydrodynamic Force $\left(f_{\mathrm{h}}\right)$ Required To Deform, Stretch, and Pull Long Linear Polystyrene Chains in Cyclohexane To Pass through Small Pores ${ }^{a}$

\begin{tabular}{ccrr}
\hline \multicolumn{2}{c}{ pore size } & $D=20 \mathrm{~nm}$ & $D=100 \mathrm{~nm}$ \\
\hline $34.3^{\circ} \mathrm{C}$ & $q_{\mathrm{c}} /(\mathrm{mL} / \mathrm{s})$ & $5.56 \times 10^{-15}$ & $3.97 \times 10^{-16}$ \\
& $f_{\mathrm{h}} / \mathrm{N}$ & $3.5 \times 10^{-14}$ & $5.3 \times 10^{-14}$ \\
$41.6^{\circ} \mathrm{C}$ & $q_{\mathrm{c}} /(\mathrm{mL} / \mathrm{s})$ & $1.85 \times 10^{-14}$ & $1.98 \times 10^{-15}$ \\
& $f_{\mathrm{h}} / \mathrm{N}$ & $1.0 \times 10^{-13}$ & $2.3 \times 10^{-13}$
\end{tabular}

${ }^{a} f_{\mathrm{h}}$ is calculated using eq 1 , and the uncertainties of $Q_{\mathrm{c}}\left(\right.$ or $\left.q_{\mathrm{c}}\right)$ and $f_{\mathrm{h}}$ are $\pm 5 \%$ and $\pm 10 \%$, respectively.

is defined as the vanishing of the second viral coefficient $\left(A_{2}\right)$, a pseudoideal state. Moreover, $\Theta$ is also a function of the chain length. $^{24}$ In an ideal solution, all the viral coefficients should approach zero, not only $A_{2}$. Therefore, the measurement of the temperature dependence of $q_{\mathrm{c}}$ might provide a better way to determine the true ideal point. The increase of $f_{\mathrm{h}}$ with the temperature in the range $T>36.5^{\circ} \mathrm{C}$ is understandable because the entropic elasticity of a polymer chain is higher when the chain expands in a better solvent. On the other hand, one has to overcome some additional segment-segment attraction force when stretching a contracted chain in a poor solvent. This is why $f_{\mathrm{h}}$ increases as the temperature decreases in the range $T<36.5^{\circ} \mathrm{C}$.

Noted that eq 2 shows that $q_{\mathrm{c}}$ should decreases as the pore size $(D)$ increases, a complicated function of $D$, different from those previous predictions of the pore size independence. ${ }^{1,2}$ To check such a discrepancy, we experimentally increased the pore size from 20 to $100 \mathrm{~nm}$ and found that $q_{\mathrm{c}}$ decreases from $1.85 \times 10^{-14}$ to $1.98 \times 10^{-15} \mathrm{~mL} / \mathrm{s}$ and from $5.56 \times 10^{-15}$ to $3.97 \times 10^{-16}$ $\mathrm{mL} / \mathrm{s}$, respectively, at 41.6 and $34.3{ }^{\circ} \mathrm{C}$. Equation 2 shows that $q_{\mathrm{c}, 20 \mathrm{~nm}} / q_{\mathrm{c}, 100 \mathrm{~nm}} \approx 3$ and 5 , respectively, for $\alpha=0.6$ (athermal) and $\alpha=0.5$ (theta), qualitatively agreeing with our experimental results $\left(\alpha \simeq 0.52\right.$ at $\left.\sim 42{ }^{\circ} \mathrm{C}\right)$. On the other hand, eq 3 shows that $f_{\mathrm{h}} \sim D^{-2+1 / \alpha}$. It means that at $T=\Theta$ (i.e., $\left.\alpha=0.5\right) f_{\mathrm{h}}$ would be independent of the pore size. Table 2 shows that at the theta state $f_{\mathrm{h}}$ is indeed less dependent on $D$, qualitatively agreeing with eq 3 .

In summary, using the critical flow rate $\left(q_{\mathrm{c}}\right)$ obtained in the ultrafiltration of polymer chains through smaller pores, we have experimentally estimated the hydrodynamic force $\left(f_{\mathrm{h}}\right)$ required to overcome the weak entropic elastic force and stretch a linear chain into a string of blobs with a size comparable to the pore size $(D)$. For linear polystyrene chains in cyclohexane at different solution temperatures, we reveal that the force required to stretch them is in the range $10-200 \mathrm{fN}$ and $q_{\mathrm{c}}$ is indeed independent of the chain length, as predicted. Therefore, it is impossible to separate linear chains with different lengths by using a given membrane with some straight pores. However, our measured values of $q_{\mathrm{c}}$ are much smaller than $k_{\mathrm{B}} T /(3 \pi \eta)$ predicted by de Gennes and Pincus. Such a discrepancy is attributed to the improper assumption that each blob made of a subchain segment is a nondraining ball. After considering a possible full hydrodynamic draining and introducing the effective length of each blob along the flow direction $\left(L_{\mathrm{e}}\right)$, we are able to qualitatively explain why $q_{\mathrm{c}}$ is $20-140$ times smaller than $k_{\mathrm{B}} T /(3 \pi \eta)$ and decreases as the pore becomes larger, especially in a good solvent. In comparison with other single-chain methods, such as a combination of tweezers and hydrodynamic force, ${ }^{26}$ our method is less precise so that the results presented here are only semiquantitative, but much cheaper, simpler, and more user-friendly.

Acknowledgment. The financial support of the Hong Kong Special Administration Region Earmarked Project (CUHK 4037/07P, 2160331) and the National Natural Scientific Foundation of China project (50773077) is gratefully acknowledged.

\section{References and Notes}

(1) De Gennes, P. G. J. Chem. Phvs. 1974, 60, 5030.

(2) Pincus, P. Macromolecules 1976, 9, 386.

(3) Rouse, P. E. J. Chem. Phvs. 1953, 21, 1272

(4) Borisov, O. V.; Darinskii, A. A.; Zhulina, E. B. Macromolecules 1995, 28, 7180

(5) Colby, R. H.; Boris, D. C.; Krause, W. E.; Dou, S. Rheol. Acta 2007, 46, 569.

(6) Jin, F.; Wu, C. Phys. Rev. Lett. 2006, 96, 237801

(7) Barrat, J. L.; Joanny, J. F. Theory of Polyelectrolyte Solutions; 1996.

(8) Lemak, A.; Lepock, J. R.; Chen, J. Z. Y. Proteins: Struct. Funct. Genet. 2003, 51, 224

(9) Perkins, T. T.; Smith, D. E.; Chu, S. Science 1997, 276, 2016.

(10) Benoit, H.; Doty, P. J. Phys. Chem. 1953, 57, 985.

(11) Bohdanecky, M. Macromolecules 1983, 16, 1483.

(12) Yamakawa, H.; Fujii, M. Macromolecules 1974, 7, 128.

(13) Bednar, J.; Furrer, P.; Stasiak, A.; Dubochet, J.; Engelman, E. H.; Bates, A. D. J. Mol. Biol. 1994, 235, 825.

(14) Frontali, C.; Dore, E.; Ferrauto, A.; Gratton, E.; Bettini, A.; Pozzan, M. R.; Valdevit, E. Biopolvmers 1979, 18, 1353.

(15) Gunari, N.; Schmidt, M.; Janshoff, A. Macromolecules 2006, 39, 2219.

(16) Zhang, W.; Zou, S.; Wang, C.; Zhang, X. J. Phvs. Chem. B 2000, 104, 10258

(17) Butt, H. J.; Kappl, M.; Mueller, H.; Raiteri, R.; Meyer, W.; Ruhe, J. Langmuir 1999, 15, 2559.

(18) Smith, S. B.; Finzi, L.; Bustamante, C. Science 1992, 258, 1122.

(19) Chu, B.; Ying, Q.; Grosberg, A. Macromolecules 1995, 28, 180.

(20) Li, J. F.; Li, W.; Hou, H.; Luo, S. Z.; Wu, C. Macromolecules 2008, 41,901 .

(21) De Gennes, P. G. Adv. Polvm. Sci. 1999, $138,91$.

(22) Rubinsein, M.; Colby, R. H. Polymer Physics; Oxford University Press: London, UK, 2003.

(23) Appelt, B.; Meyerhoff, G. Macromolecules 1980, 13, 657.

(24) Melchionna, S.; Bernaschi, M.; Fyta, M.; Kaxiras, E.; Succi, S. Phvs. Rev. E 2009, 79, 030901.

(25) Saeki, S.; Kuwahara, N.; Konno, S.; Kaneko, M. Macromolecules 1973, 6, 246

(26) Saleh, O. A.; McIntosh, D. B.; Pincus, P.; Ribeck, N. Phvs. Rev. Lett. 2009, 102, 068301. 